\title{
Estrategias motivacionales para fortalecer habilidades de emprendimiento en estudiantes de bachillerato
}

\section{Motivational strategies to strengthen entrepreneurship skills in high school students}

DOI: $10.46932 / \mathrm{sfjdv} 2 \mathrm{n} 5-115$

Received in: Oct 1st, 2021

Accepted in: Dec 30th, 2021

\section{Marjorie Magdalena Jara Jiménez}

Ingeniera en Gestión Empresarial. Magister en Administración de Empresas. Docente de Emprendimiento, Unidad Educativa Mocache, Mocache, Los Ríos, Ecuador.

Dirección: Mocache km. 1 1 1/2 vía a Quevedo.

E-mail: clari_mar22@hotmail.com

\section{Liliana Isabel Flores Anchundia}

Ingeniera en Administración Financiera. Magister en Contabilidad y Auditoría. Egresada en Maestría de Gestión Educativa. Docente en el Área de Contabilidad, Unidad Educativa José María Velasco Ibarra,

Buena Fe, Los Ríos, Ecuador.

Dirección: calle arcadio fuente y transversal sin nombre junto al cementerio general.

E-mail: lili_isaldu@hotmail.es

Victoria María Jiménez Ramos

Licenciada en Ciencias de la Educación. Master en Ciencias de La Educación. Docente de la Unidad Educativa La Maná, Cotopaxi, Ecuador.

Dirección: av. 19 de mayo y Jaime Roldos, La Maná.

E-mail: victoriamariajimenez@gmail.com

María Isabel Cabrera Briones

Ingeniera en Gestión Empresarial. Maestra en Psicología Educativa.

Dirección: Quevedo, san camilo, calle México y la D.

E-mail: mariacabrera79@yahoo.es

\section{RESUMEN}

El presente trabajo de investigación se desarrolló teniendo como objetivo general proponer un manual de estrategias motivacionales para el fortalecimiento de las habilidades de emprendimiento de los estudiantes de bachillerato.

La metodología se basó en un enfoque cuantitativo de tipo descriptivo proyectivo, con diseño de tipo no experimental, en donde se utilizó como técnica la entrevista, siendo el instrumento de recolección de datos el cuestionario, este estuvo dirigido a un grupo de estudio, integrado por 76 estudiantes, el mismo que estuvo constituido por 41 ítems, fue validado a través del juicio de cinco expertos y la confiabilidad se realizó a través del coeficiente del Alfa de Cronbach, que arrojó un nivel de fiabilidad de0,842. Así mismo se utilizaron herramientas estadísticas como Excel y SPSS para el procesamiento de datos.

Los resultados permitieron concluir que: hay un porcentaje un poco bajo de estudiantes que no se sienten motivados en las horas de clases. Un porcentaje bajo de estudiantes que no tienen confianza en sí mismos al momento de expresar sus opiniones. Existe un grupo bajo de estudiantes se sienten incomodos estudiando de manera virtual, lo que hace que no disfruten de sus clases. Un grupo de estudiantes no son empáticos con sus compañeros, ni se muestran asertivos cuando se comunican con ellos. Un grupo de 
estudiantes no tienen facilidad para relacionarse con sus compañeros. Un grupo de estudiantes que no les gusta trabajar en equipo ni incorporarse a trabajar en equipo. Un grupo de estudiantes no asumen sus propias conclusiones ni tienen capacidad para decidir rápidamente y cuando toman decisiones y otro grupo que no son justos, optimistas, no tienen carisma ni autocontrol de sus emociones. En conclusión, se comprobó que es necesario el empleo de un manual de técnicas que permita el desarrollo de estrategias motivacionales para el fortalecimiento de las habilidades de emprendimiento de los estudiantes.

Palabras claves: Estrategias motivacionales, habilidades personales, habilidades sociales, habilidades de dirección, habilidades de emprendimiento.

\begin{abstract}
The present research work was developed with the general objective of proposing a manual of motivational strategies for strengthening the entrepreneurial skills of high school students.

The methodology was based on a quantitative projective descriptive approach, with a non-experimental design, where the interview was used as a technique, the questionnaire being the data collection instrument, this was directed to a study group, composed of 76 students, which consisted of 41 items, was validated through the judgment of five experts and the reliability was made through the Cronbach's Alpha coefficient, which yielded a reliability level of 0.842. Likewise, statistical tools such as Excel and SPSS were used for data processing.

The results allowed us to conclude that: there is a slightly low percentage of students who do not feel motivated during class hours. A low percentage of students who do not have confidence in themselves when expressing their opinions. There is a low group of students who feel uncomfortable studying virtually, which makes them not enjoy their classes. A group of students are not empathic with their peers, nor are they assertive when communicating with them. A group of students do not have the facility to relate to their peers. A group of students who do not like to work in a team or join teamwork. A group of students do not assume their own conclusions or have the ability to decide quickly and when they make decisions and another group that are not fair, optimistic, they do not have charisma or self-control of their emotions. In conclusion, it was evidenced that it is necessary to use a manual of motivational strategies to strengthen the entrepreneurial skills of students.
\end{abstract}

Keywords: Motivational strategies, personal skills, social skills, management skills, entrepreneurship skills.

\title{
1 INTRODUCCION
}

La educación para el emprendimiento identifica el impacto que tiene el desarrollo del espíritu emprendedor en desarrollo de la economía, esto se debe a la motivación que tienen las personas para empezar sus negocios y generar empleo. Los emprendimientos son mecanismos claves para el incremento de la economía y el desarrollo en el ámbito innovador de un lugar determinado. El hecho de crear y fundar nuevas empresas se asocia con la generación que busca la consecución de empleo, para poder genera riqueza, el aumento de la competitividad y la puesta en marcha del desarrollo tecnológico (Vallmitjana i Palau, 2014)

En lo nacional la situación del análisis de la actividad emprendedora se halla actualmente en su fase inicial y con grandes perspectivas de aumento exponencial en los próximos cuatro años. Algunas 
universidades cuentan con grupos de investigación que se orientan los análisis a la actividad emprendedora y a demás disponen de mecanismos que dan soporte a los nuevos emprendedores en sus proyectos de tipo empresarial. (Delgado M 2020).

La condición de vida actual a raíz de la pandemia ha demostrado lo importante que representa el desarrollo de las habilidades de emprendimiento en los estudiantes, que le permiten la vinculación de los saberes obtenidos en el proceso educativos, además de la oportunidad de generar ingresos y mejorar su condición de vida y la de sus familiares.

Dentro del contexto educativo se orienta al estudiante en el área de emprendimiento más por diferentes factores no se estimula el desarrollo de las habilidades que le permitirán abrirse paso en esta actividad de una manera activa y próspera perdiendo el miedo a la situación y convirtiéndose en un ente futurista en pro del progreso y desarrollo, el docente orienta el quehacer educativo en base a la planificación que se encuentra estructurada en el material de lectura que ofrece el Gobierno nacional, pero es importante que este plantee estrategias motivacionales que favorezcan el desarrollo de las habilidades de emprendimiento de los estudiantes.

La base del problema radica en que el entorno que rodea al estudiante es hostil y por temor a enfrentar realidades como la crítica destructiva, la burla, y comentarios de alguien tipo despectivo u otras situaciones que no sólo desmoraliza también pueden resultar perturbadoras y frustrantes para el estudiante en formación y para el desempeño correcto de su emprendimiento; la no estimulación de las habilidades de emprendimiento como las habilidades sociales, habilidades de desarrollo, habilidades de competencias en el estudiantes generan dudas, miedo, inquietud, desinterés desgano y estos factores contribuyen a que el estudiante no tome la decisión de emprender, desvalorizando su autoestima elevando su temor a no cumplir con las expectativas de la sociedad insensible que le rodea.

A través de lo expuesto es importante destacar que, las instituciones educativas cuentan con la participación activa de los docentes, pero el temor de la participación de los estudiantes en el aula de clase, evidencia la falta de fortalecimiento de habilidades de emprendimiento, por este motivo el desarrollo de la investigación formula el siguiente problema ¿Qué estrategias motivacionales debe proponer un manual para el fortalecimiento de las habilidades de emprendimiento de los estudiantes de bachillerato? Las interrogantes específicas son, ¿Cuáles son las características de las habilidades personales como la iniciativa, confianza, motivación y comunicación que generan emprendimientos en los estudiantes de bachillerato?, asimismo, ¿Cómo se pueden examinar las habilidades sociales mediante las relaciones personales y trabajo en equipo de los estudiantes de bachillerato?, y finalmente, ¿Qué características presentan las habilidades de dirección como la toma de decisiones y el liderazgo que generan el emprendimiento de los estudiantes de bachillerato. ¿Qué aspectos debe contener un manual para el fortalecimiento de las habilidades de emprendimiento de los estudiantes de bachillerato? 
A continuación, se explican las teorías que sustentas la variable habilidades de emprendimiento, entre ellas tenemos la teoría del capital humano de Schultz (1961, como se citó en Pérez-Fuentes \& Castillo-Loaiza, 2016) en la que se sostiene que la formación del conocimiento y la experiencia, las cuales representan un valor más alto en relación con el éxito económico y empresarial frente a otros, por lo tanto se enfoca en estudiar aspectos de acuerdo con las capacidades y habilidades de las personas que se han adquirido durante su formación.

La teoría económica de Mark Casson (1982, como se citó en Terán-Yépez \& Guerrero-Mora, 2020) destaca más a las cualidades personales e innatas de las personas; según esta teoría, el nivel alcanzado por el emprendimiento se encuentra condicionado por las cualidades personales que posee el emprendedor, ya de estas se deriva el nivel de alcance que tienen las ofertas de un emprendimiento específico.

Las habilidades de emprendimiento según Ocampo \& López (2020) conciernen al proceso de creación de destreza, en donde se asocia a las competencias administrativas y actitudes empresariales, con el objetivo de saber aprovechar todo los que está alrededor, esto implica saber evaluar los recursos y los riesgos.

El manejo de las habilidades personales es fundamental para desarrollar el cuidado personal, gestionar y dirigir a los demás y lograr resultados satisfactorios. En este contexto, el emprendedor debe ser capaz de optimizar sus habilidades y facilitar su comportamiento mediante factores motivacionales, sentimientos adecuadamente constituidos y actitudes que le faciliten la identificación de situaciones con el mayor control y dominio de las emociones. (Ferro, 2019).

De acuerdo con Díaz-García, et al. (2020) la capacidad de trabajo en equipo en todo ámbito organizacional, aptitud para hallar procesos que resuelvan conflictos y tomar acciones prontas en la toma de decisiones, capacidades comunicativas para convencer, liderar e informar, responsable ante iniciativas, creativos e innovador para crear oportunidades, responder a cada una de las necesidades y situaciones de forma óptima y tener objetivos claros que garantizan bueno resultados y orientaciones.

La segunda dimensión de las habilidades de emprendimiento son las sociales, que según Tapia y Cubo (2017) representan el conjunto de comportamientos o conductas sociales que forman parte del individuo que son adquiridas en el transcurso de la vida, mediante escenarios representativos en la vida del ser humano, estas habilidades están compuestas por la aplicación de normas sociales o valores interpersonales que permiten la facilidad de interacción en cualquier situación.

Para Flores, et al (2016) la autoestima es la base para el desarrollo efectivo de las habilidades sociales que construyen y reflejan la personalidad de las personas; esta representa el valor que se tiene sobre sí mismas. Es fundamental porque dirige las acciones y la interacción de las personas con el exterior, además, genera expectativas en los demás pudiendo ser estas, negativas o positivas. 
La tercera dimensión denominada habilidades directivas según son todos aquellos conocimientos adquiridos en la labor de gestión o competencias para dirigir y emplear correctamente los recursos y herramientas necesarias para cumplir con las metas de la organización.

En los sistemas educativos se requiere de líderes capacitados para gestiones que se adaptan al entorno de trabajo con ideas creativas, por otro lado, brinden seguridad para afrontar metas en la organización y no sólo brinden seguridad, sino que creen proyecto para mejorar la calidad y la productividad.

Las habilidades Ascón \& García (2019) directivas se consideran necesarias para el desempeño del líder, es decir que se necesitan estas habilidades para organizar, medir y crear protocolos, para mejorar la comunicación, resolver conflictos, analizar, y poseer habilidades técnicas.

La variable estrategias de motivación se sustenta en teorías que amplían las vías de comprensión de su impacto en el desarrollo de las habilidades de los estudiantes. La primera es la teoría de modificación de las conductas de Skinner (/0000) la cual explica que toda persona reacciona de acuerdo al estímulo que recibe del ambiente externo. (Lima \& Araújo, 2018)

La segunda teoría es la de Equidad de Stacey Adams (1963) la cual sostiene que en un ambiente colectivo resulta alentador para todos los miembros el predominio de los valores institucionales, entre los que se menciona el trato justo, la equidad de oportunidades y valoración del desempeño personal.

La investigación principalmente se basa en la teoría sociocultural de Vygotsky (1981), que atribuye mayor importancia a la interacción social, como principal factor del aprendizaje cognoscitivo. Según esta teoría el aprendizaje en la etapa escolar debe ser autónomo, pero a la vez guiado a partir de herramientas que faciliten el trayecto por el camino del aprendizaje. (Santana, et al., 2021)

En la definición de la variable estrategias de motivación es preciso considerar que una realidad en el aula es la prioridad del cumplimiento de las actividades de acuerdo a lo planificado y por ello, en ciertas ocasiones se evita otorgar minutos de motivación inicial, empezando de manera directa con la explicación de tema.

Gamificación: para Pertegal \& Lorenzo (2019) es una herramienta metodológica o técnica de aprendizaje diseñada en para mejorar los procesos de formación académico, por medio de un conjunto de recurso creados precisamente para ayudar a mejorar el rendimiento escolar en los estudiantes (p. 554).

Estrategias vivenciales: para Lara (2018) las vivenciales son todas aquellas experiencias, trabajo, conocimiento, comportamientos, acciones, valores, principio y aprendizajes anteriores, que se aplican en cualquier proceso de formación, en una conversación, en la ejecución de proyectos y en los trabajos (p. 48). 
Según Abregú \& Galve (2016) las técnicas empleadas por medio de las vivencias involucran buenas relaciones sociales, interactivas y prácticas, se basan en principios y normas practicas reales que genera ánimo, participación y trabajo colaborativo.

Estrategias afectivas: las estrategias afectivas, como hablarse así mismo, hacerse una autovaloración de sí mismo positivamente, visualizar oportunidades para emplear métodos correctos y cumplirlos y un conjunto de acciones para mejorar sus emociones y las actividades de aprendizajes, tienen como objetivos la motivación intrínseca que aumente lo efectos relacionados con el mejoramiento de los aprendizajes.

Los estudiantes que se autogestionan para mejorar los aprendizajes aplican estrategias funcionales y significativas para comprender cualquier problema de la vida real en todo ámbito, se instruyen de tan forma que ellos saben correctamente cuando aplicar estrategias enseñadas y diseñadas para por ellos.

\section{METODOLOGIA}

La presente investigación fue descriptiva, con un enfoque cuantitativo, de tipo no experimental con un diseño proyectivo aplicado, para Guevara, Verdesoto, \& Castro Nelly (2020) esta investigación tiene la finalidad de relatar rasgos esenciales de fenómenos de estudio, usando características fundamentales de conjuntos homogéneos de fenómenos, utilizando razonamientos que permitan la estructura o conducta del dicho fenómeno. Por otra parte, Cadena (2017), señala que el enfoque cuantitativo es aquel donde se recogen datos para realizar el análisis respecto al objeto de estudio, por otro lado, Rodríguez (2017), manifiesta que las investigaciones cuantitativas estudian acontecimientos observables.

Por lo tanto, en esta investigación se recogió datos, que permitieron conocer las características del objeto de estudio para aplicar las estrategias motivacionales adecuadas que fortalecerán las habilidades de emprendimiento de los estudiantes, y al mismo tiempo mejorará la educación de los estudiantes.

Fue de diseño proyectivo o propositivo por cuanto se permitió la manipulación de al menos una variable (Pastrano. 2019) en este caso la variable fática y el análisis que la misma posee en la variable teórica solamente permite la diferencia de los experimentos en el grado de seguridad o confiabilidad que pueda tomarse en cuenta sobre las equivalencias iniciales de los grupos. Para el desarrollo de este diseño metodológico se aplicó la prueba piloto para el nivel de confiabilidad y el diagnóstico con las encuestas dirigidas a los estudiantes el esquema que se utiliza para el desarrollo de la investigación es el siguiente: 


\section{RESULTADOS}

OE1: Describir las características de las habilidades personales como la iniciativa, confianza, motivación y comunicación que generan emprendimientos en los estudiantes de bachillerato.

Tabla 1: Características de la iniciativa, según la dimensión de las habilidades de personales.

\begin{tabular}{|c|c|c|c|c|c|}
\hline & \multicolumn{5}{|c|}{ CASI } \\
\hline & \multicolumn{3}{|c|}{ NUNCA A VECES SIEMPRE } & \multicolumn{2}{|c|}{ SIEMPRETotal } \\
\hline & $\mathrm{N}^{\circ} \%$ & $\mathrm{~N}^{\circ} \%$ & $\%$ & $\mathrm{~N}^{\circ} \%$ & $\mathrm{~N}^{\circ} \%$ \\
\hline $\begin{array}{l}\text { 1. ¿Toma la iniciativa para responder a las actividades durante la hora de } \\
\text { clases? }\end{array}$ & $1621,1 \%$ & $61925,0 \% 16$ & $21,1 \%$ & 2532 & $\% 76100,0 \%$ \\
\hline 2. ¿Se siente motivado para participar activamente en clases? & $79,2 \%$ & $2938,2 \% 16$ & $21,1 \%$ & 2431 & $\% 76100,0 \%$ \\
\hline 3. ¿Genera sus propias ideas? & $56,6 \%$ & $2330,3 \% 16$ & $21,1 \%$ & 3242 & $\% 76100,0 \%$ \\
\hline 4. ¿Se compromete responsablemente con sus obligaciones? & $79,2 \%$ & $1215,8 \% 23$ & $30,3 \%$ & 3444 & $\% 76100,0 \%$ \\
\hline 5. ¿Asume los riesgos? & $810,5 \%$ & $62026,3 \% 24$ & $31,6 \%$ & 2431 & $\% 76100,0 \%$ \\
\hline
\end{tabular}

Fuente: Cuestionario dirigido a estudiantes.

Los datos obtenidos en la dimensión de habilidades personales en el indicador iniciativa, el 46,00\% entre a veces y nunca los estudiantes toman la iniciativa para responder a las actividades durante la hora de clase, también el 47,30\% entre, a veces y nunca se sienten motivados para participar en la hora de clases, en tanto que un $36,80 \%$ en a veces y nunca generan sus propias ideas, asimismo un 25,00 a veces y nunca se compromete responsablemente con sus obligaciones, seguido de un $36,80 \%$ entre a veces y nunca asume los riesgos. En tal sentido los resultados evidenciaron que hay un porcentaje un poco bajo de estudiantes que no se sienten motivados en las horas de clases, lo que hace que sientan temor de generar ideas y tomar la iniciativa para dar respuestas a las actividades planteadas en su hora clase y por tanto no se atreven a asumir riesgos.

Tabla 2: Características de la confianza, según la dimensión de las habilidades de personales.

\begin{tabular}{|c|c|c|c|c|c|c|c|}
\hline & NUNCA & \multicolumn{2}{|c|}{$\begin{array}{c}\text { A } \\
\text { VECES }\end{array}$} & \multicolumn{2}{|c|}{ CASI } & SIEMPRE & Total \\
\hline & $\mathrm{N}^{\circ} \%$ & $\mathrm{~N}^{\circ}$ & $\%$ & $\mathrm{~N}^{\circ}$ & $\%$ & $\mathrm{~N}^{\circ}$ & $\mathrm{N}^{\circ}$ \\
\hline $\begin{array}{l}\text { 6. ¿Tiene confianza en sí mismo a la hora de expresar sus } \\
\text { opiniones? }\end{array}$ & $1114,5 \%$ & 18 & $23,7 \%$ & 27 & $35,5 \%$ & $2026,3 \%$ & $76100,0 \%$ \\
\hline 7. ¿Se siente cómodo/a al pedir ayuda a sus compañeros? & $1621,1 \%$ & 19 & $25,0 \%$ & 25 & $32,9 \%$ & $1621,1 \%$ & $76100,0 \%$ \\
\hline $\begin{array}{l}\text { 8. ¿Respeta las preferencias y las necesidades de sus } \\
\text { compañeros? }\end{array}$ & $1114,5 \%$ & 18 & $23,7 \%$ & 11 & $14,5 \%$ & $3647,4 \%$ & $76100,0 \%$ \\
\hline 9. ¿Cree que sus opiniones e ideas son importantes? & $1114,5 \%$ & 13 & $17,1 \%$ & 16 & $21,1 \%$ & $3647,4 \%$ & $76100,0 \%$ \\
\hline
\end{tabular}

Fuente: Cuestionario dirigido a estudiantes.

En la tabla 2, se observa que en el indicador confianza de las habilidades personales un 38,20\% entre a veces y nunca los estudiantes tienen confianza en sí mismos al momento de expresar sus opiniones, para continuar el 46,00\% entre nunca y a veces se sienten cómodos al pedir ayuda a sus compañeros, en tanto un $38,10 \%$, entre a veces y nunca respetan las preferencias y las necesidades de sus compañeros seguidamente de un $31,50 \%$ entre nunca y a veces cree que sus opiniones e ideas son importantes. Esto 
quiere decir que existe un porcentaje bajo de estudiantes que no tienen confianza en sí mismos al momento de expresar sus opiniones, por tanto, no piden ayuda a sus compañeros, porque creen que sus opiniones no son importantes.

Tabla 3: Características de la motivación, según la dimensión de las habilidades de personales

\begin{tabular}{|c|c|c|c|c|c|c|c|c|c|c|}
\hline & \multicolumn{10}{|c|}{ CASI } \\
\hline & \multicolumn{2}{|c|}{ NUNCA } & \multicolumn{2}{|c|}{ A VECES } & \multicolumn{2}{|c|}{ SIEMPRE } & \multicolumn{2}{|c|}{ SIEMPRE } & \multicolumn{2}{|r|}{ Total } \\
\hline & $\mathrm{N}^{\circ}$ & $\%$ & $\mathrm{~N}^{\circ}$ & $\%$ & $\mathrm{~N}^{\circ}$ & $\%$ & $\mathrm{~N}^{\circ}$ & $\%$ & $\mathrm{~N}^{\circ}$ & $\%$ \\
\hline 10. ¿Siente incomodidad estudiando virtualmente? & 21 & $27,6 \%$ & 24 & $31,6 \%$ & 17 & $22,4 \%$ & 14 & $18,4 \%$ & 76 & $100,0 \%$ \\
\hline 11. ¿Disfruta de sus clases? & 10 & $13,2 \%$ & 17 & $22,4 \%$ & 25 & $32,9 \%$ & 24 & $31,6 \%$ & 76 & $100,0 \%$ \\
\hline $\begin{array}{l}\text { 12. ¿Tiene problemas de conectividad para recibir sus } \\
\text { clases? }\end{array}$ & 10 & $13,2 \%$ & 34 & $44,7 \%$ & 21 & $27,6 \%$ & 11 & $14,5 \%$ & 76 & $100,0 \%$ \\
\hline $\begin{array}{l}\text { 13. ¿Las actividades que realiza su docente son } \\
\text { monótonas? }\end{array}$ & 12 & $15,8 \%$ & 31 & $40,8 \%$ & 24 & $31,6 \%$ & 9 & $11,8 \%$ & 76 & $100,0 \%$ \\
\hline 14. ¿Los docentes muestran interés por su aprendizaje? & 8 & $10,5 \%$ & 10 & $13,2 \%$ & 10 & $13,2 \%$ & 48 & $63,2 \%$ & 76 & $100,0 \%$ \\
\hline 15. ¿Son reconocidos sus logros por el docente? & 11 & $14,5 \%$ & 15 & $19,7 \%$ & 28 & $36,8 \%$ & 22 & $28,9 \%$ & 76 & $100,0 \%$ \\
\hline 16. ¿Recibe clases de manera lúdica? & 9 & $11,8 \%$ & 21 & $27,6 \%$ & 30 & $39,5 \%$ & 16 & $21,1 \%$ & 76 & $100,0 \%$ \\
\hline 17. ¿Se siente obligado a estudiar? & 43 & $56,6 \%$ & 15 & $19,7 \%$ & 11 & $14,5 \%$ & 7 & $9,2 \%$ & 76 & $100,0 \%$ \\
\hline 18. ¿Siente que sus clases son difíciles? & 12 & $15,8 \%$ & 28 & $36,8 \%$ & 28 & $36,8 \%$ & 8 & $10,5 \%$ & 76 & $100,0 \%$ \\
\hline
\end{tabular}

Fuente: Cuestionario dirigido a estudiantes.

Los datos obtenidos en la tabla 3, en la dimensión de habilidades sociales, el indicador motivación, un así como también un 35,50\% a veces y nunca los estudiantes disfrutan de sus clases, seguidamente de un 56,60\% entre a veces y nunca creen que las actividades que realiza el docente son monótonas, así mismo un 34,30\% entre nunca, casi nunca y casi siempre, manifestaron que no son reconocidos sus logros por el docente, por otro lado el 39,40\% a veces y nunca recibe clases de manera lúdica. Los resultados indicaron que existe un grupo bajo de estudiantes se sienten incomodos estudiando de manera virtual, lo que hace que no disfruten de sus clases y consideran que las actividades que realiza el docente son monótonas, así como también sienten que sus logros no son reconocidos por parte de los docentes.

Tabla 4: Características de la comunicación, según la dimensión de las habilidades de personales.

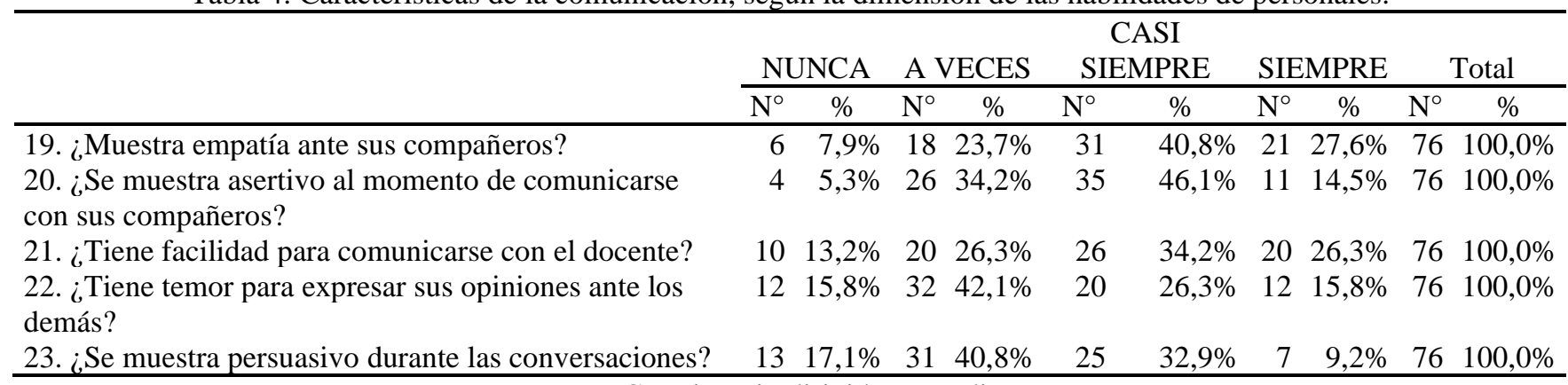

Fuente: Cuestionario dirigido a estudiantes.

La tabla 4 del indicador comunicación de las habilidades personales, indica que un $31,60 \%$ a veces y nunca muestra empatía ante sus compañeros, así mismo un 39,40\% a veces y nunca se muestra asertivo al momento de comunicarse con sus compañeros, seguidamente de un 39,50\% entre a veces y nunca, no 
tienen facilidad para comunicarse con el docente, también un 57,90\% a veces y nunca, tiene temor de expresar sus opiniones ante los demás, así como 57,90\% entre a veces y nunca se muestra persuasivo durante las conversaciones. Esto quiere decir que un grupo de estudiantes no son empáticos con sus compañeros, ni se muestran asertivos cuando se comunican con ellos, e incluso presentan dificultades para comunicarse con sus docentes por ello sienten temor al expresar sus opiniones ante los demás y demuestran no ser persuasivos durante las conversaciones.

OE2: Examinar las habilidades sociales mediante las relaciones personales y trabajo en equipo para de los estudiantes de bachillerato.

Tabla 5: Características de las relaciones personales, según la dimensión de las habilidades sociales.

\begin{tabular}{|c|c|c|c|c|c|c|c|c|c|c|}
\hline & \multicolumn{10}{|c|}{ CASI } \\
\hline & \multicolumn{2}{|c|}{ NUNCA } & \multicolumn{2}{|c|}{ A VECES } & \multicolumn{2}{|c|}{ SIEMPRE } & \multicolumn{2}{|c|}{ SIEMPRE } & \multicolumn{2}{|r|}{ Total } \\
\hline & $\mathrm{N}^{\circ}$ & $\%$ & $\mathrm{~N}^{\circ}$ & $\%$ & $\mathrm{~N}^{\circ}$ & $\%$ & $\mathrm{~N}^{\circ}$ & $\%$ & $\mathrm{~N}^{\circ}$ & $\%$ \\
\hline $\begin{array}{l}\text { 24. ¿Tiene facilidad para relacionarse con sus } \\
\text { compañeros? }\end{array}$ & 14 & $18,4 \%$ & 26 & $34,2 \%$ & 27 & $35,5 \%$ & 9 & $11,8 \%$ & 76 & $100,0 \%$ \\
\hline 25. ¿Es cortés con sus docentes y compañeros? & 6 & $7,9 \%$ & 11 & $14,5 \%$ & 19 & $25,0 \%$ & 40 & $52,6 \%$ & 76 & $100,0 \%$ \\
\hline 26. ¿Se muestra tolerante con los demás? & 14 & $18,4 \%$ & 12 & $15,8 \%$ & 27 & $35,5 \%$ & 23 & $30,3 \%$ & 76 & $100,0 \%$ \\
\hline 27. ¿Se interesa por los problemas de sus compañeros? & 9 & $11,8 \%$ & 18 & $23,7 \%$ & 36 & $47,4 \%$ & 13 & $17,1 \%$ & 76 & $100,0 \%$ \\
\hline
\end{tabular}

Fuente: Cuestionario dirigido a estudiantes.

Los datos obtenidos en la tabla 5, del indicador relaciones personales correspondiente a la dimensión de Habilidades sociales, un 52,70\% entre a veces y nunca tienen facilidad para relacionarse con sus compañeros, así mismo un 34,20\% entre a veces y nunca se muestra tolerante con los demás, en tanto que un 35,50\% entre a veces y nunca se interesa por los problemas de sus compañeros. Los resultados reflejaron que hay un grupo de estudiantes no tienen facilidad para relacionarse con sus compañeros, así como también en ocasiones no muestran tolerancia hacia los demás, ni se interesan por sus problemas.

Tabla 6: Características del trabajo en equipo, según la dimensión de las habilidades sociales.

\begin{tabular}{|c|c|c|c|c|c|c|c|c|c|c|}
\hline & \multicolumn{2}{|c|}{ NUNCA } & \multicolumn{2}{|c|}{ A VECES } & \multicolumn{2}{|c|}{ CASI SIEMPRE } & \multicolumn{2}{|c|}{ SIEMPRE } & \multicolumn{2}{|r|}{ Total } \\
\hline & $\mathrm{N}^{\circ}$ & $\%$ & $\mathrm{~N}^{\circ}$ & $\%$ & $\mathrm{~N}^{\circ}$ & $\%$ & $\mathrm{~N}^{\circ}$ & $\%$ & $\mathrm{~N}^{\circ}$ & $\%$ \\
\hline $28 . i$ & 11 & $14,5 \%$ & 19 & $25,0 \%$ & $\overline{16}$ & 21 , & 30 & $39,5 \%$ & 76 & $100,0 \%$ \\
\hline 29. ¿Se inco & 8 & $10,5 \%$ & 18 & $23,7 \%$ & 27 & $35,5 \%$ & 23 & $30,3 \%$ & 76 & $100,0 \%$ \\
\hline 30. ¿Siente placer al ayudar a sus com & 7 & $9,2 \%$ & 11 & $14,5 \%$ & 30 & $39,5 \%$ & 28 & $36,8 \%$ & 76 & $100,0 \%$ \\
\hline 31. ¿Se muestra colaborador con los demás? & 6 & $7,9 \%$ & 17 & $22,4 \%$ & 23 & $30,3 \%$ & 30 & $39,5 \%$ & 76 & $100,0 \%$ \\
\hline
\end{tabular}

Fuente: Cuestionario dirigido a estudiantes.

En la tabla 6 correspondiente al indicador trabajo en equipo de la dimensión habilidades sociales, se evidencia un 39,40\%, entre a veces y nunca les gusta trabajar en equipo, por otro lado el 34,20\% entre a veces y nunca los estudiantes se incorporan a los grupos de trabajo, seguidamente de un $23,70 \%$ entre a veces y nunca siente placer al ayudar a sus compañeros, también un 30,20\%, entre a veces y nunca se muestra colaborador con los demás. Estos resultados mostraron que hay un grupo de estudiantes que no 
les gusta trabajar en equipo ni incorporarse a trabajar en grupos, tampoco sienten agrado al ayudar a los demás por lo que se muestran poco colaboradores con sus compañeros.

OE3: Analizar las habilidades de dirección como la toma de decisiones y el liderazgo para fortalecer el emprendimiento de los estudiantes de bachillerato.

Tabla 7: Características de la toma de decisiones, según la dimensión de las habilidades de dirección.

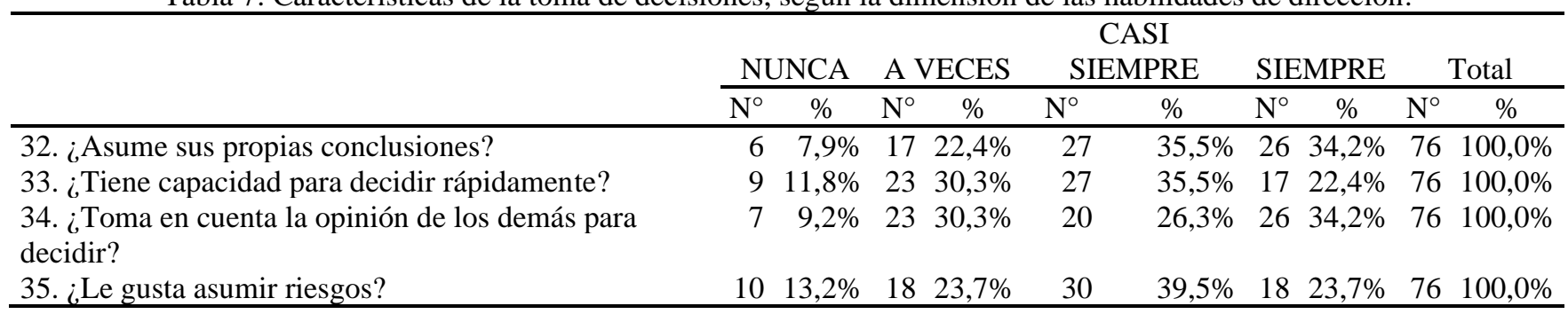

Fuente: Cuestionario dirigido a estudiantes.

Los datos que contienen la tabla 7 muestran el indicador toma de decisiones de las habilidades de dirección, donde el 30,30\% entre a veces y nunca asume sus propias conclusiones, así mismo un 42,10\% entre a veces y nunca tienen capacidad para decidir rápidamente, por otra parte, un $39,50 \%$ entre a veces y nunca toma en cuenta la opinión de los demás para decidir, en tanto que un 36,80\% entre a veces y nunca le gusta asumir riesgos. Esto quiere decir que un grupo de estudiantes no asumen sus propias conclusiones ni tienen capacidad para decidir rápidamente y cuando toman decisiones lo hacen sin considerar la opinión de los demás ni asumir riesgos.

Tabla 8: Características del liderazgo, según la dimensión de las habilidades de dirección.

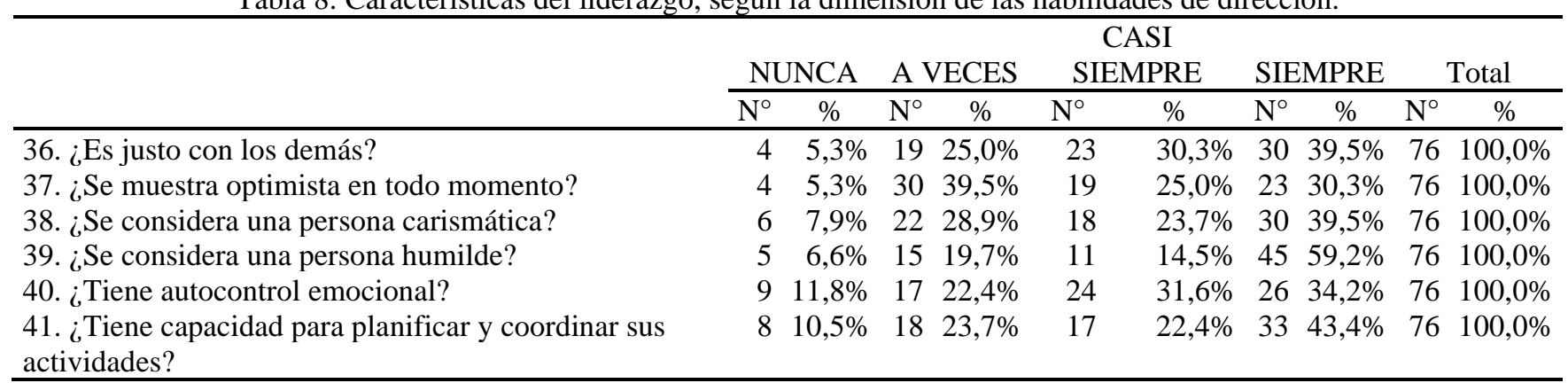

Fuente: Cuestionario dirigido a estudiantes.

La tabla 8 que contiene datos del indicador Liderazgo de la dimensión habilidades de dirección, muestra que el 30,20\% entre a veces y nunca los estudiantes no son justos con los demás, seguidamente de un $44,70 \%$ entre a veces y nunca se muestran optimistas en todo momento, por otro lado el $36,80 \%$ entre a veces y nunca se consideran carismáticos, así mismo el $26,30 \%$ entre a veces y nunca tiene autocontrol emocional, en tanto un $34,20 \%$, entre a veces y nunca los estudiantes tienen capacidad para planificar y coordinar sus actividades. Los resultados reflejaron que un grupo de estudiantes no son justos, 
optimistas, no tienen carisma ni autocontrol de sus emociones por tanto no tienen capacidad para planificar y llevar una buena organización de sus actividades.

OE4: Identificar los aspectos debe contener un manual para el fortalecimiento de las habilidades de emprendimiento de los estudiantes de bachillerato.

Tabla 9: Aspectos debe contener un manual para el fortalecimiento de las habilidades de emprendimiento.

\begin{tabular}{ccl}
\hline \multicolumn{1}{c}{ DIMENSIÓN } & TABLA & \multicolumn{1}{c}{ ELEMENTO } \\
\hline Habilidades personales & $\mathrm{N}^{\circ} 4$ & $\begin{array}{l}\text { Bajo nivel de auto concepto personal. } \\
\text { Bajo nivel de auto estima y poca valoración a su capacidad } \\
\text { El temor a enfrentarse a resolver problemas. }\end{array}$ \\
& $N^{\circ} 6$ & Falta de empatía ante situaciones adversas de sus pares y de ellos mismos \\
Habilidades sociales & $\mathrm{N}^{\circ} 7$ & $\begin{array}{l}\text { Escasa práctica de relaciones interpersonales. } \\
\text { Escaso nivel de comunicación con sus pares }\end{array}$ \\
& & Escaso control de emociones ante discusiones y peleas entre compañeros.
\end{tabular}

Desconocimiento de la importancia del emprendimiento como alternativa laboral.

Fuente: Cuestionario dirigido a estudiantes.

Los datos que reflejan la tabla 9 muestran los aspectos que debe contener un manual para el fortalecimiento de habilidades de emprendimiento de los estudiantes de Bachillerato.

\section{DISCUSION}

En el primer objetivo se buscó: Describir las características de las habilidades personales como la iniciativa, confianza, motivación y comunicación que generan emprendimientos en los estudiantes de bachillerato. En la tabla 1 se evidencian los resultados de las características de la iniciativa, según la dimensión de las habilidades de personales. En tal sentido, se observó que hay un porcentaje un poco bajo de estudiantes que no se sienten motivados en las horas de clases, lo que hace que sientan temor de generar ideas y tomar la iniciativa para dar respuestas a las actividades planteadas en su hora clase y por tanto no se atreven a asumir riesgos. En este contexto, las habilidades personales de los docentes y estudiantes juegan un rol importante durante la clase. Por tanto, existe la necesidad de construir habilidades comunicativas que lleven a los estudiantes a comprender la importancia de las relaciones interpersonales e interacción con los demás para el uso adecuado de la comunicación.

Estos resultados se asimilan a un estudio realizado por Pérez (2016) quien mediante una "propuesta metodológica para la caracterización de pautas emprendedoras en materia de emprendimiento universitario", los resultados descriptivos revelaron que la necesidad motivacional de logro alcanzó una media de 17.59 en el primer curso y 17.78 en cuarto curso, por consiguiente, la necesidad de planificación alcanzó una media de 17.59 en el primer curso. Se concluyó que el emprendimiento en los estudiantes es 
una habilidad poco fomentada, que merece fortalecerse dentro de la formación académica a partir de actividades centradas en la acción y logro de objetivos.

En la tabla 2, se observan las características de la confianza, según la dimensión de las habilidades de personales. En la cual, se muestra un porcentaje bajo de estudiantes que no tienen confianza en sí mismos al momento de expresar sus opiniones, por tanto, no piden ayuda a sus compañeros, porque creen que sus opiniones no son importantes. La confianza es importante en las habilidades personales porque los profesores desarrollan habilidades de comunicación para establecer relaciones eficaces de enseñanzaaprendizaje.

Estos resultados se asemejan con el estudio de Ruíz \& Biencinto (2019) quienes desarrollaron un estudio correlacional sobre la motivación e identidad de los licenciados chinos de español y la propuesta didáctica de mejora para el ELE en China", los resultados indicaron que existen diferencias significativas entre la motivación del aprendizaje, la confianza y la dimensión personal ( $\mathrm{p}=.000)$. En cuanto a la intensidad de la motivación el $67.2 \%$ alcanza una intensidad media, seguid del $17.6 \%$ cuya intensidad de motivación es alta y el $15.3 \%$ de baja.

En la tabla 3, se encuentran las características de la motivación, según la dimensión de las habilidades de personales. En referencia a las características de la motivación, como indicador de las habilidades personales, entendida como el estado interno que activa, dirige y mantiene la conducta de la persona hacia metas o fines determinados; además es el impulso que mueve a la persona a realizar determinadas acciones y persistir en ellas para su culminación, se observó que existen grupos considerables que tienen una baja motivación, al no sentirse cómodos estudiando de manera virtual, ya que no disfrutan de estas clases, especialmente porque no tienen una buena conectividad, sumándose a ello la monotonía del trabajo docente, quienes por la recargada labor no valoran los logros de los estudiantes, por lo cual la mayoría de estudiantes, si van a estudiar lo hacen por obligación.

Los hallazgos presentados, se asocia a los encontrados por Zevallos (2016) quien en su estudio "influencia de la motivación en el aprendizaje motor de estudiantes", encontró que existen diversos niveles de motivación en los estudiantes y estos se producen a partir de las expectativas y recompensas externas como internas.

En la tabla 4, se encuentran las características de la comunicación, según la dimensión de las habilidades de personales. En la cual se observó un grupo de estudiantes no son empáticos con sus compañeros, ni se muestran asertivos cuando se comunican con ellos, e incluso presentan dificultades para comunicarse con sus docentes por ello sienten temor al expresar sus opiniones ante los demás y demuestran no ser persuasivos durante las conversaciones. En efecto, el ambiente de aprendizaje y la comunicación juega un importante rol en el desarrollo de habilidades de emprendimiento de los estudiantes, por lo cual se hace necesario que los docentes tengan en cuenta aquellos factores que 
garanticen una motivación adecuada en los estudiantes, durante el desarrollo de sus actividades académicas. Estos resultados se asemejaban al estudio de Peralta, (2018), en su investigación realizada referente al tema en discusión, concluye que las estrategias de motivación favorecen el desarrollo de las habilidades de emprendimiento. En conclusión, se determinó que las estrategias de motivación contribuyen en las habilidades de emprendimiento, como la creatividad, las habilidades de planificación, la capacidad de relaciones sociales y personales.

En el segundo objetivo se buscó: Examinar las habilidades sociales mediante las relaciones personales y trabajo en equipo para de los estudiantes de bachillerato del Distrito 12D03 MocacheQuevedo, 2021. Con respecto a este objetivo se evaluaron los siguientes indicadores:

En la tabla 5, las características de las relaciones personales, según la dimensión de las habilidades sociales. En la cual, los resultados reflejaron que hay un grupo de estudiantes no tienen facilidad para relacionarse con sus compañeros, así como también en ocasiones no muestran tolerancia hacia los demás, ni se interesan por sus problemas. Con base en los datos teóricos las habilidades sociales las habilidades sociales construyen y reflejan la personalidad de las personas; esta representa el valor que se tiene sobre sí mismas. Es fundamental porque dirige las acciones y la interacción de las personas con el exterior, además, genera expectativas en los demás pudiendo ser estas, negativas o positivas. Este estudio se relaciona con el de Mónico, Carvalho, \& Nejati, (2021), quienes realizaron un estudio sobre "la educación para el emprendimiento y su influencia en las intenciones y la motivación emprendedora de los estudiantes de bachillerato". Para lo cual, se encontró que la educación emprendedora no tuvo un efecto directo sobre la habilidad emprendedora de los estudiantes. Se concluyó que, estabilidad y significancia en el modelo estructural de las habilidades personales frente a la motivación emprendedora no fue significativa.

En la tabla 6 se encuentran las características del trabajo en equipo, según la dimensión de las habilidades sociales. Estos resultados mostraron que hay un grupo de estudiantes que no les gusta trabajar en equipo ni incorporarse a trabajar en grupos, tampoco sienten agrado al ayudar a los demás por lo que se muestran poco colaboradores con sus compañeros, aunque la mayoría de los estudiantes si colaboran con sus compañeros de forma incondicional. Es decir que, las habilidades sociales no se pueden definir fácilmente como una sola cosa, sino que consisten en una constelación de habilidades y comportamientos. Por su parte estas habilidades permiten al ser humano alcanzar sus objetivos sociales, es decir, gestionar adecuadamente sus relaciones interpersonales, lo que puede repercutir en otros ámbitos de la vida. Suelen basarse en la gestión de la comunicación y las emociones.

Este estudio se asemeja con el de Pérez (2019), quien investigó sobre las "habilidades sociales en estudiantes de secundaria de una institución educativa particular del distrito de San Juan de Lurigancho". Entre los resultados se tuvo que tuvo un $44.44 \%$ corresponde a un nivel "Deficiente" y un $14.81 \%$ 
corresponde a un nivel "Bajo", con respecto a las habilidades sociales. Se concluyó que en el trabajo en equipo las habilidades sociales son de suma relevancia para fortalecer la comunicación.

En el tercer objetivo se buscó: Analizar las habilidades de dirección como la toma de decisiones y el liderazgo para fortalecer el emprendimiento de los estudiantes de bachillerato. En referencia a lo descrito se muestran a continuación los indicadores evaluados:

En la tabla 7, se encuentran las características de la toma de decisiones, según la dimensión de las habilidades de dirección. En los resultados se evidenció que existe un grupo de estudiantes no asumen sus propias conclusiones ni tienen capacidad para decidir rápidamente y cuando toman decisiones lo hacen sin considerar la opinión de los demás ni asumir riesgos. En relación a las habilidades de dirección, se entiende que estas son aquellas que aumentan la productividad de los estudiantes, sino que también da un buen ejemplo en el desarrollo de conocimientos. A través de ello se pueden detectar las fortalezas de los estudiantes y alentarlos a desarrollar sus habilidades. Según lo analizado en la investigación se pudo conocer que un grupo de estudiantes no son justos, optimistas, no tienen carisma ni autocontrol de sus emociones por tanto no tienen capacidad para planificar y llevar una buena organización de sus actividades.

Estos resultados se relacionan con los encontrados en el estudio de Herrera, (2020) llevó a cabo el estudio sobre "El rendimiento y la motivación para el emprendimiento de los estudiantes en el bachillerato técnico". En el cual se indica que los docentes tienen habilidades necesarias para fortalecer la motivación para el emprendimiento de los estudiantes. Se reflejaron diferencias en el aprendizaje, presentándose un incremento del esfuerzo para actividades relacionadas con las tutorías en línea, uso de herramientas de colaboración y comunicación para el emprendimiento. Por lo tanto, se expresa que existe significancia entre las habilidades de dirección como la toma de decisiones y el liderazgo.

En la tabla 8, se encuentran las características del liderazgo, según la dimensión de las habilidades de dirección. Los resultados reflejaron que un grupo de estudiantes no son justos, optimistas, no tienen carisma ni autocontrol de sus emociones por tanto no tienen capacidad para planificar y llevar una buena organización de sus actividades. Con referencia a las habilidades de dirección y el liderazgo se pudo analizar que los líderes dentro de las escuelas deben trabajar para comprender las necesidades y habilidades de desarrollo personal de los estudiantes, así como las brechas de conocimiento. Esto puede ayudar a crear una cultura de aprendizaje y responsabilidad, predicando con el ejemplo para demostrar cómo el estudiante puede mejorar su capacidad.

Este estudio se relaciona con Valenzuela, Muñoz, \& Montoya (2018) quienes investigaron sobre las "estrategias motivacionales efectivas en profesores en formación” cuyas dimensiones de análisis fueron la motivación hacia el aprendizaje, motivación con la relación de tareas y motivación como entretención. Los resultados obtenidos revelaron que el 20,49\% de las estrategias aplicadas corresponden 
a la motivación del aprendizaje, el $12,18 \%$ se orientan a las estrategias de enseñanza y el $67.34 \%$ son estrategias orientadas a motivar la atención de los estudiantes. Se concluyó que, los líderes en las escuelas deben asegurarse de que una cultura de investigación, innovación y creatividad esté en el corazón de la organización, y deben buscar comentarios y datos regulares sobre el desempeño de los estudiantes en relación con los cambios.

Los resultados que se obtuvieron fueron basados en una investigación de tipo descriptivo, de tipo no experimental, con diseño proyectivo, donde se propuso un manual de estrategia motivacionales para fortalecer habilidades de emprendimiento en estudiantes de bachillerato, sin embargo queda la interrogante ¿La aplicación del manual de estrategias motivacionales para estudiantes de bachillerato fortalecerá las habilidades personales, sociales y de dirección de los estudiantes?, la misma que daría paso a una nueva investigación.

\section{CONCLUSIONES}

En conclusión, es importante considerar el desarrollo de las habilidades personales como la iniciativa, confianza, motivación y comunicación que generan emprendimientos en los estudiantes de bachillerato. Es de suma importancia desarrollar la comunicación porque tiene un impacto significativo en el bienestar y capacidad para tener éxito académico.

$\mathrm{Al}$ examinar las habilidades sociales mediante las relaciones personales y trabajo en equipo se fortalecen las habilidades de los estudiantes de bachillerato. En este caso, trabajar en equipo en diferentes asignaciones minimiza las cargas de trabajo para todos los estudiantes, al compartir ideas y responsabilidades, lo que permite que sean críticos al momento de desarrollar las tareas designadas.

Al analizar las habilidades de dirección como la toma de decisiones y el liderazgo se podrá fortalecer el emprendimiento de los estudiantes de bachillerato. Se aplica porque la decisión de colaboración permite a los estudiantes interactuar entre sí en un proyecto. Esta interacción es una gran oportunidad de aprendizaje para adquirir habilidades que no tenían y volverse más experimentados en el emprendimiento.

Por último, se concluye que, un manual para el fortalecimiento de las habilidades de emprendimiento de los estudiantes de bachillerato debe contener actividades que fortalezcan las habilidades personales, sociales y de dirección en los estudiantes de forma que se logre superar dificultades de aprendizajes. 


\section{REFERENCIAS}

Abregú, L., \& Galve, J. (2016). La estrategia didáctica vivencial aplicada en la enseñanza-aprendizaje de la salud en el trabajo. Education yPsychology I+D+i and Editorial EOS, 8(2), 1228 - 2010. doi:https://doi.org/10.25115/ejrep.v8i22.1413

Ascón, J., \& García, M. (2019). Habilidades directivas. Estrategias de formación turística en la Universidad de La Habana. 2(1), 1-20. doi: https://doi.org/10.33262/exploradordigital.v2i1.326

Balcázar, A. (2021). The educational camp as a setting for strengthening social skills. Retos(41), 143-153. Cadena, P., Rendón, R., Aguilar, J., Salinas, E., Cruz, F., \& Sangerman, D. (2017). Métodos cuantitativos, métodos cualitativos o su combinación en la investigación: un acercamiento en las ciencias sociales. Revista mexicana de ciencias agrícolas, 8(7), 1603-1617. México.

Caro, L. (2019). Técnicas e instrumentos para la recolección de datos. 7. Obtenido de http://148.202.167.116:8080/xmlui/handle/123456789/2801

Díaz-García, I., Almerich, G., Suárez-Rodríguez, J., \& Orellana, N. (2020). The relationship between ICT competences, ICT use and learning approaches in university students of education. Revista de Investigación Educativa, 38(20), 549-566. doi:https://doi.org/10.6018/rie.409371

Escudero, C., \& Cortez, L. (2018). Tecnicas y metodos para la investigacion cientifica. Machala: Editorial Utmach. Obtenido de http://repositorio.utmachala.edu.ec/bitstream/48000/12501/1/Tecnicas-yMetodoscualitativosParaInvestigacionCientifica.pdf

Ferro, J. (2019). Trabajo en equipo para mejorar la calidad laboral. Universidad Católica de Colombia. Bogotá : Universidad Católica de Colombia. Obtenido de https://repository.ucatolica.edu.co/bitstream/10983/24011/1/trabajo\%20en\%20equipo\%20para\%20mejo rar\%201a\%20calidad\%20laboral.pdf

Flores, E., García, M., Calsina, W., \& Yapuchura, A. (2016). The social skills and the interpersonal communication of the students of the national university of altiplano - puno. Comunicación: Revista de Investigación en Comunicación y Desarrollo, 7(2), 5-14.

Guevara, G., Verdesoto, A., \& Castro Nelly. (2020). Metodologías de investigación educativa (descriptivas, experimentales, participativas, y de investigación-acción). Recimundo, 164.

Lara, F. (2018). Experiential strategies to promote values in first grade students in the area of religious education of the educational institution José Contreras Cabrera de Pomacucho. unheval, 45-68. Doi:oai:172.16.0.151:unheval/2924

Lima, F., \& Araújo, E. (2018). Condicionamento operante de Skinner: a influência do desempe-nho no estágio de operações na selva da siesp. Revista Agulhas Negras, 2(2), 62-70. Obtenido de http://ebrevistas.eb.mil.br/aman/article/view/1874/1513

Ocampo, C., \& López, Y. (2020). Habilidades emprendedoras: importancia, evolución y sub temas emergentes. Una revisión de literatura. Económicas CUC, 52-73. doi:http://dx.doi.org/10.17981/econcuc.42.1.2021.Org.4 
Otzen, T., \& Manterola , C. (2017). Técnicas de Muestreo sobre una Población a Estudio. International journal of morphology., 35(1), 227-232. Chile. Obtenido de https://scielo.conicyt.cl/scielo.php?pid=S0717-95022017000100037\&script=sci_arttext

Paredes-Pérez, M., Cárdenas-Tapia, V., \& Palomino-Crispin, A. (2021). Habilidades directivas y planificación estratégica en una universidad peruana. Gaceta Científica, 7(1), 17-22. Obtenido de https://doi.org/10.46794/gacien.7.1.1061

Pastor, F. (2019). Población y muestra. Pueblo continente. 30(1), 245-247. Perú. Obtenido de http://200.62.226.189/PuebloContinente/article/view/1269/1099

Pérez-Fuentes, D., \& Castillo-Loaiza, J. (2016). Human capital, theories and methods: importance of the health variable. Econ. soc. territ, 16(52), 651-673. Obtenido de http://www.scielo.org.mx/scielo.php?script=sci_arttext\&pid=S1405-

$84212016000300651 \& \operatorname{lng}=$ es\&tlng=es.

Pertegal, L., \& Lorenzo, G. (2019). Gamification in the classroom through the TIC. INFAD, 553-562. doi:10.17060/ijodaep.2019.n1.v3.1535

Rivadeneira, E. (2017). Lineamientos teóricos y metodológicos de la investigación cuantitativa en ciencias sociales. . In Crescendo, 8(1), 115-121. Obtenido de https://doi.org/10.21895/incres.2017.v8n1.11

Rodriguez, M. (2017). Lineamientos teóricos y metodológicos de la investigación cuantitativa en ciencias sociales. In Crescendo, 8(1), 115-121. Obtenido de file:///C:/Users/PERSONAL/Downloads/DialnetLineamientosTeoricosYMetodologicosDeLaInvestigacio-6042332.pdf

Santana, R., Izaguirre, R., \& Reyes, J. (2021). Influence of the sociocultural context in the development of historical thought. Opuntia Brava, 13(1), 232-245. Obtenido de http://200.14.53.83/index.php/opuntiabrava/article/view/1382

Tapia-Gutiérrez, C., \& Cubo-Delgado, S. (2017). Relevant Social Skills: Perceptions of Multiple Educational Actors. Magis. Revista Internacional de Investigación en Educación, 133-148. doi:doi: 10.11144/Javeriana.m9-19.hsrp

Terán-Yépez, E., \& Guerrero-Mora, A. (2020). Entrepreneurship theories: A critical review of the literature and future research suggestions. Espacios, 41(7), 1-7. Obtenido de http://www.revistaespacios.com/a20v41n07/a20v41n07p07.pdf

Ventura, J. (2017). Population or sample?: A necessary difference. Revista Cubana de Salud Pública., 43(4). Obtenido de http://scielo.sld.cu/scielo.php?script=sci_arttext\&pid=S0864-34662017000400014 